\title{
"It's not you, it's us": A hopeful reflection on the tensions of uniting participatory health research and evidence-based medicine
}

\author{
Krystyna Kongats
}

\section{University of Alberta}

Nearly 15 years ago, Health Canada identified public involvement as a key challenge of governance in the years ahead, stating that citizen engagement would assist the resolution of most major national challenges. ${ }^{1}$ Participatory approaches to research in primary health care have increased over the past decade because of their potential to address inequities in health. ${ }^{2,3}$ Participatory health research is an approach that engages citizens in identifying health problems, participating in all aspects of the research process and in designing solutions. ${ }^{4}$ Considering such developments, the focus of this commentary is to spark a conversation on the tensions of integrating citizen participation within primary health care; and consequently, to consider the merging of two distinct paradigms: participatory health research and evidence-based medicine. The commentary will conclude by exploring the unique role tensions play in learning and transformation. As citizen participation in research on primary health care is gradually turning into official policy, it is important to discuss these tensions moving forward to capitalize on the value of participation in the health sector. Uncovering these tensions is only a first step in opening a space for conversations on uniting participatory health research and evidence-based medicine.

While there are many reasons for engaging citizens in research on primary health care, here I present three key rationales. ${ }^{3}$ First, citizens have lived experience of their health conditions that can benefit researchers and practitioners. Second, it is a right for citizens to be involved in publically funded research that affects their health. Third, public involvement has the potential to strengthen the relevance, impact and accountability of research and service delivery in primary health care. These arguments demonstrate that the integration of citizen participation within primary health care presents new opportunities for addressing inequities in health. However, it also presents new challenges.
First, a central tenant of the participatory health research paradigm is the value placed on different ways of knowing, including experiential knowledge. ${ }^{4}$ Experiential knowledge comes from the lived experience of citizens who have valuable knowledge of their health conditions and contexts, which is inherently subjective. This subjectivity is a source of tension within an evidence-based medicine model that considers best evidence to be objective and value-free. ${ }^{5}$

Evidence-based medicine assumes that maintaining a distance between direct experience and interpretation increases reliability. ${ }^{5}$ In contrast, participatory health research emphasizes that the shorter the distance there is between lived experience and how it is interpreted, the more relevant and accurate the resulting knowledge is likely to be. ${ }^{5}$

Second, participatory health research brings attention to power differentials. Power plays an important role in whose voices are acknowledged. Within a participatory health research paradigm, equal weight is given to lay and professional knowledge. ${ }^{4}$ This conflicts with how knowledge is perceived within an evidence-based medicine model that abides to a hierarchy of evidence. For example, evidencebased medicine places randomized control trials at the top of this hierarchy and expert opinion at the bottom. ${ }^{6}$ Within this pyramid of evidence, there is no mention of the contribution of lay knowledge.

Third, participatory health research is locally situated. The knowledge generated through participation is context-specific, grounded in the reality of daily life and work. ${ }^{7}$ This is in contrast to the value that is placed on generalizable knowledge within an evidence-based model. ${ }^{8}$ What we are left with is a "generalizability paradox" ${ }^{2}$ whereby increasing the relevance and specificity of research questions to local contexts decreases the generalizability of the study findings. 
The tensions presented in this commentary demonstrate a few challenges in meaningfully engaging citizens in primary health care research and practice as two worlds come together, each with their own historical structures and assumptions. ${ }^{8}$ Ultimately, if we view these tensions as a driving force for learning rather than obstacles, we can bring together multiple perspectives and create new ways of thinking and understanding. ${ }^{8}$ It is these new perspectives that initiate transformations in the way research questions are developed, in how services are provided and in the sustainability of our health care system.

Improving the quality and impact of primary health care and reducing health inequities is about transformation. While bringing forth exciting changes and possibilities, such transformation is not always comfortable as it calls upon us to question our current practices. ${ }^{8}$ It is my hope that by presenting these tensions I can contribute to sparking conversations on how we can find common ground in our differences. If we are to really address health inequities and proposed policy statements on meaningful public involvement within primary health care, finding common ground and moving forward through transformation are essential.

\section{References}

1. Secretariat CC. Health Policy and Communications Branch, Health Canada. The Health Canada policy toolkit for public involvement in decision making. Ottawa: Minister of Public Works and Government Services Canada; 2000.

2. Cargo M, Mercer SL. The value and challenges of participatory research: strengthening its practice. Annu Rev Public Health. 2008;29:325-350.

3. Boote J, Baird W, Beecroft C. Public involvement at the design stage of primary health research: a narrative review of case examples. Health Policy. 2010;95(1):10-23.

4. Ledwith M, Springett J. Participatory practice. Bristol: The Policy Press; 2010.

5. Beresford P. Developing the theoretical basis for service user/survivorled research and equal involvement in research. Epidemiol Psychiatr Soc. 2005;14(1):4-9.

6. Evans D. Hierarchy of evidence: a framework for ranking evidence evaluating healthcare interventions. J Clin Nurs. 2003;12(1):77-84.

7. International Collaboration for Participatory Health Research. Position paper 1: what is participatory health research [online]. Berlin: International Collaboration for Participatory Health Research; 2013 [last update unknown; cited 2014 Feb 2]. Available from: http://tinyurl.com/qygp3y4.

8. Aas M. Towards a reflection repertoire: using a thinking tool to understand tensions in an action research project. Educ Action Res. 2014 Feb 3 [Epub ahead of print].

\section{Krystyna Kongats}

Krystyna Kongats is a first year health promotion PhD student at the School of Public Health, University of Alberta. She is part of the Ecological and Participatory Approaches to Health Research (Eco-PATH) lab where she studies the democratization of science and citizen participation in health research and practice. She is also an executive committee member of the International Collaboration for Participatory Health Research. This summer she is looking forward to trying her hand at urban community gardening. 